\title{
Time Course of Localization for a Repeatedly Flashing Stimulus Presented at Perisaccadic Timing
}

\author{
Junji Watanabe, ${ }^{1}$ Taro Maeda, ${ }^{2}$ and Susumu Tachi ${ }^{1}$ \\ ${ }^{1}$ Graduate School of Information Science and Technology, The University of Tokyo, Tokyo, 113-8656 Japan \\ ${ }^{2}$ NTT Communication Science Laboratories, NTT Corporation, Atsugi, 243-0198 Japan
}

\section{SUMMARY}

When a light spot is flashed repeatedly during saccade, a dotted line called a phantom array (PA) is perceived. When light spots are placed on a vertical line and the flashing pattern is changed quickly during saccade, the flashing pattern is expanded to a spatial pattern by the effect of eye movement, and a two-dimensional image is perceived. The authors have proposed a visual information presentation method based on this phenomenon. A crucial issue in information presentation by this method is the time relation between the perceived PA and the eye movement. Consequently, this study examines the time relation between the perception and the eye movement. The time course of perceived position for repeatedly flashing stimulus is investigated by presenting light spots in the vertical position. It is found that the locus of the perceived light spot starts to curve at the start of saccade in the direction opposite to the saccade, and returns to the vertical direction when the saccade ends. It differs from the locus of the light spot which is expected in accordance with cancellation theory. (C) 2005 Wiley Periodicals, Inc. Syst Comp Jpn, 36(9): 77-86, 2005; Published online in Wiley InterScience (www.interscience.wiley.com). DOI 10.1002/scj.10690

Key words: saccade; display; spatial localization error; cancellation theory; phantom array.

\section{Introduction}

In visual information presentation methods with twodimensional spread, the scanning process, such as a CRT display, in which the information is presented by moving a light spot at high speed has mostly been used. There is also a device, as shown in the left panel of Fig. 1, in which a physical motion is performed and the two-dimensional information is presented by using light spots in a row.

On the other hand, the following phenomenon exists. Light spots are fixed in a row. When the flashing pattern is changed at high speed while the observer is in saccade, the flashing pattern is expanded into a spatial pattern by the eye movement, and a two-dimensional image is perceived. It is thus possible to present information efficiently with little
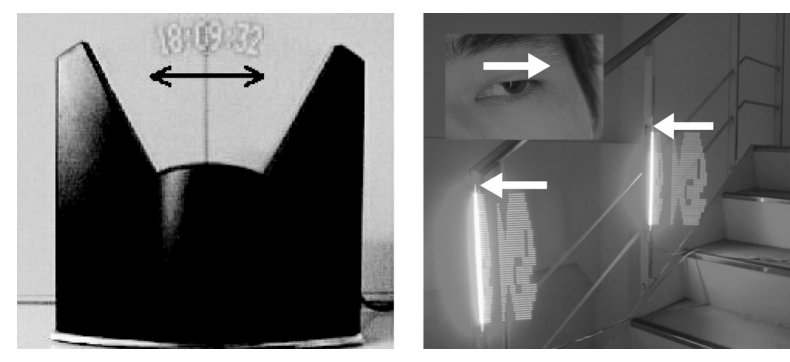

Fig. 1. Information display device based on moving light array and based on eye movement.

(C) 2005 Wiley Periodicals, Inc. 
space and energy without moving the light spot itself, but by using eye movement, as in the right panel of Fig. 1. The authors have been proposing a visual information presentation method (saccade-based display, SD) based on this principle [1].

The perception characteristics differ between the saccade state and the gaze state. Consequently, the design theory for SD must be composed on the basis of the perception characteristics during saccade. There has been a report in which a light spot is repeatedly flashed during saccade and the direction of motion of the light spot is reported, in an attempt to analyze the time course relation between the light spot perception position and the eye movement [2].

However, the subjective perception of the direction of motion of the light spot includes individual differences. It will also be affected by uncontrollable factors such as the attention of the observer [3]. Consequently, in this study, the light spot stimulus is flashed in the vertical direction so that the time course of the light spot perception position is placed by the vertical position, in an attempt to analyze the time course relation between the perception of the repeatedly flashing light spot and the eye movement without utilizing subjective report.

\section{Spatial Perception Characteristics during Saccade}

This section outlines the visual information presentation method utilizing saccade. The relation between this study and past studies concerning the light spot perception in saccade is discussed.

\subsection{Visual information presentation method based on saccade}

In $\mathrm{SD}$ the information is presented as follows. The eye movement of the observer is not measured. By flashing the light spot as the target of saccade, the information provider tries to induce saccade (with a maximum movement velocity of 300 to $500 \%$ s [4]). Then, by flashing the light spot according to the saccade, the information is displayed [1]. For reliable information presentation, the light spot row is flashed at high speed from $100 \mathrm{~ms}$ before to $100 \mathrm{~ms}$ after the saccade.

It is generally suggested that perception is suppressed during saccade and that the resolution of perception is degraded. There is also a report, however, that the suppression is smaller for stimuli with a high spatial frequency, as presented in SD [5]. In fact, it has been verified that when the background intensity is low and the contrast of the stimulus with the background is sufficient, there is little degradation of visual resolution [1]. Fujita and Amami reported that information such as characters can be perceived during saccade [6].

\subsection{Perception of light spot with instantaneous presentation}

In connection with light spot perception characteristics during saccade, Honda presented a study of the error of spatial localization [7, 8]. Spatial localization error refers to the phenomenon in which, when a flash light spot is presented during saccade, its position is localized with an error that has a certain tendency. Figure 2 shows the concept of the phenomenon, in which the magnitude of the localization error is the vertical axis, and the time (ms) is the horizontal axis. The solid line represents the eye position, and the dotted line is the time course of the localization error magnitude.

Generally, when the light spot is presented before saccade, the localization error is in the same direction as the saccade. If it is presented during the saccade, the error localization gradually changes. When it is presented after the saccade, the localization error is in the opposite direction to the saccade. The absolute localization error is maximized if the presentation is near the start or end of the saccade. This phenomenon can be accounted for by the cancellation theory between the internal information of eye position and the retinal information [8].

\subsection{Perception of repeatedly flashing light spot}

Hershberger reported the following phenomenon. When a light spot is repeatedly flashed during saccade at a frequency of $120 \mathrm{~Hz}$, a dotted line is perceived, appearing successively in the opposite direction to the saccade, as shown in Fig. 3 [9]. This is called a phantom array (PA).

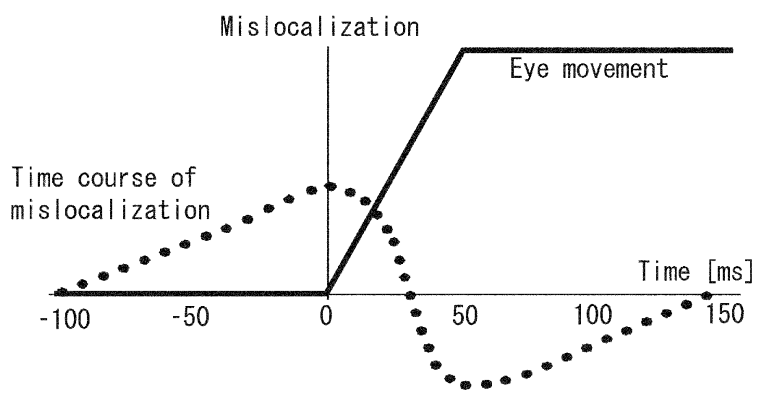

Fig. 2. Time course of mislocalization before, during, and after a saccade. 


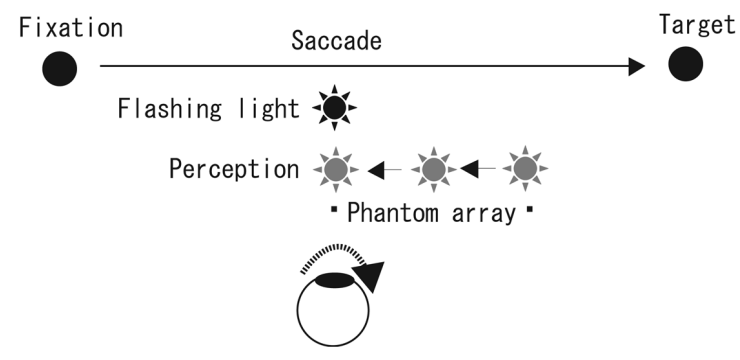

Fig. 3. Perception of phantom array.

If the perceived locus for the repeatedly flashing light spot is the curve that is obtained by connecting the perceived position for the flash light spots, as shown in Fig. 2, the PA should be moved to the same direction as the saccade immediately before and after the saccade. Such a subjective perception, however, has not been reported in the experiments in this paper, or in the study by Hershberger and colleagues $[9,10]$.

\subsection{Features of image perceived by SD}

The image perceived by SD differs from the image on the retina generated by eye movement in terms of size and position. Specifically, the image perceived by a saccade of $10^{\circ}$ differs from the image of $10^{\circ}$ projected on the retina as in Fig. 4. Its size is approximately $5^{\circ}$, which is approximately half the retinal image as in the lower side of Fig. 4 (it sometimes happens, as in the figure, that the perceived image covers the light spot array). Hershberger's group also

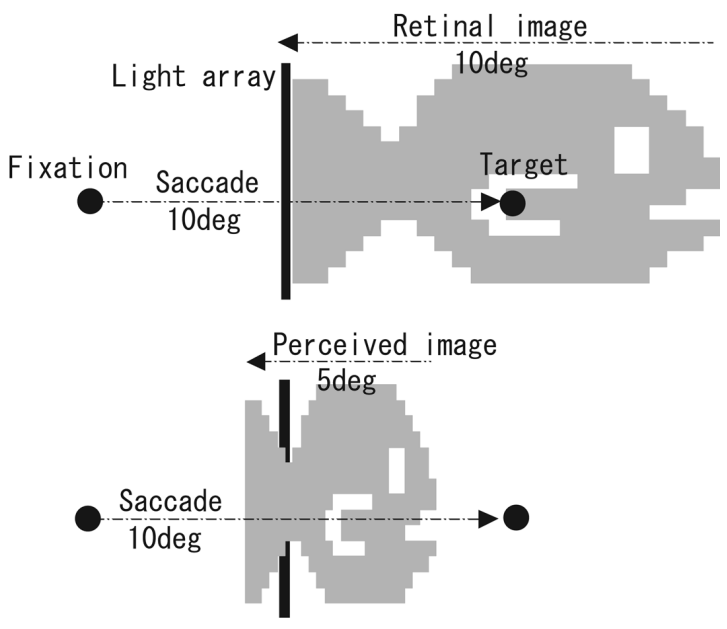

Fig. 4. Retinal image (top) and perceived image (bottom). reported that the perceived PA has approximately half the size of the eye movement [10]. Essentially, it is interpreted that the two-dimensional image perceived by SD is equivalent to the vertical arrangement of PA.

\section{Time Relation between PA Perception and Eye Movement}

In order to determine the flash timing of the light spot array in the information presentation based on $\mathrm{SD}$, it is crucial to know the time relation between PA and eye movement. In the experiment by Hershberger's group, the light spot is repeatedly flashed only during saccade. They do not discuss the time relation between the perceived PA and the eye movement. Consequently, the light spot is flashed in perisaccadic period in this study, and the time relation between the perception and the eye movement is examined by a psychophysical experiment.

\subsection{Procedure of experiment}

The fixation point (FP), the target point (TP), and the light spot (S) are placed as shown on the left side of Fig. 5. Sixty-four LEDs are arranged vertically as S. S is placed at the center between FP and TP. The distance between the right eye of the subject and $\mathrm{S}$ is set as $150 \mathrm{~cm}$ and the distance between FP and TP is set as $8^{\circ}$ or $12^{\circ}$ in terms of the view angle. The height of FP, TP, and the center of $\mathrm{S}$ are set at the same height as the eyes of the subject.

The right side of Fig. 5 shows the time chart of the experiment. As the first step, FP is flashed at random for 1 to $2 \mathrm{~s}$, and the subject looks toward FP. Then, FP is left off. After $200 \mathrm{~ms}$, TP is on for $10 \mathrm{~ms}$. The subject performs a horizontal saccade toward TP. In the process, after $62 \mathrm{~ms}$ from TP being off, $\mathrm{S}$ is flashed successively from the top. The flash is $2 \mathrm{~ms}$ per spot, or $128 \mathrm{~ms}$ in all (throughout this 128 -ms period, only one of LEDs is on). The gap of $200 \mathrm{~ms}$ is set in order to induce the express saccade, in which the

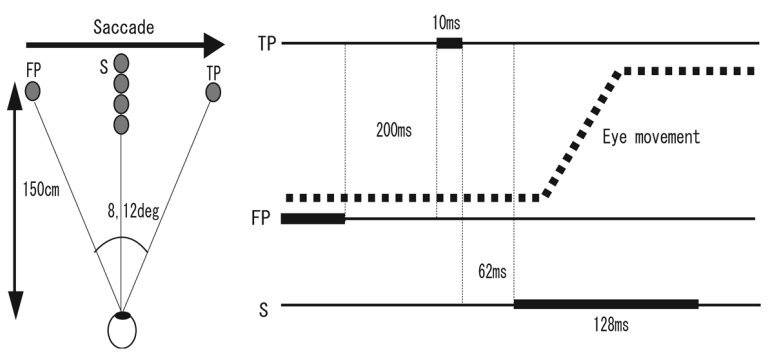

Fig. 5. Outline of the experiment and time chart. 
latency is especially stable in time [11], so that stable experimental conditions are realized.

The locus of the light spot which is perceived by the subject in saccade, always has the shape of the sigmoid function, which is interpreted as a result of vertical expansion with the time axis of PA as on the left of Fig. 6. In the experiment, the eye movement of the subject is measured. The subject is also instructed to indicate with a laser pointer the "start point," where the perceived light spot locus starts to curve in the horizontal direction, and the "end point," where the locus returns to the straight line (cross on the left of Fig. 6). The indicated position is recorded on a section plane containing the focus point, target, and light spot array. The position is read on the basis of the light of the pointer. The read value is not reported to the subject.

The start and the end of the flashing light spot array were measured in parallel to the eye movement. Based on these records, the time relation between the period for which the light spot was on at the position reported by the subject and the onset/offset of the saccade can be determined. In the experiment, the magnitude of the saccade is set in two ways, as $8^{\circ}$ and $12^{\circ}$.

\subsection{Experimental device}

A red LED (diameter $0.25^{\circ}$ and $60 \mathrm{~cd} / \mathrm{m}^{2}$ ) was used for the fixation point (FP) and the target point (TP). A green LED (diameter $0.2^{\circ}$ and $20 \mathrm{~cd} / \mathrm{m}^{2}$ ) was used for the repeatedly flashing light spot array (S). The vertical length of $S$ was $12.8^{\circ}$. A PIC16F877 single-chip microprocessor was used for the flashing control of the LED. The subject covered the left eye using the eye-band and indicated the start and end points of the perceived light spot locus. The head of the subject was fixed on the chin rest. The eye movement was measured with $600-\mathrm{Hz}$ sampling, using an EMR-600 produced by NAC Co. The experiment was conducted in a completely dark room $(0.011 \mathrm{x})$. The subjects

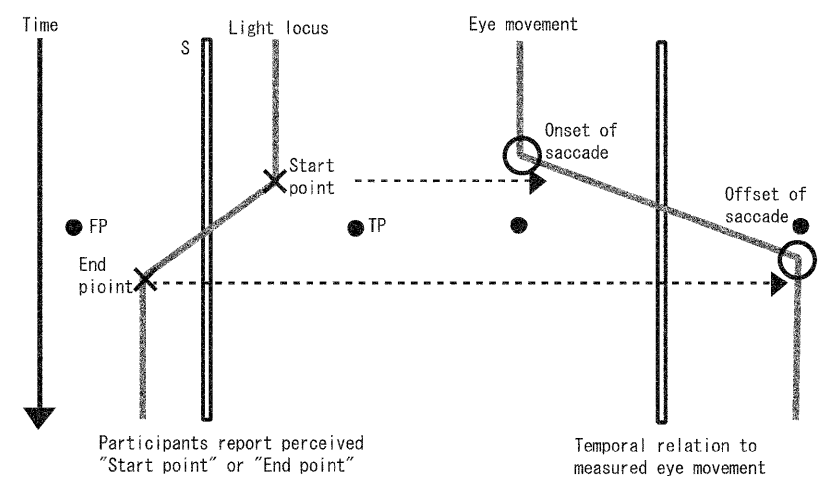

Fig. 6. Perceived image (left) and eye position (right). were four men in their twenties who had visual acuities of at least 0.6 with correction.

\subsection{Preliminary experiment}

In this experiment, the light spot array was flashed successively from the top to the bottom so that the time course of the PA spread in the horizontal direction in space was expanded into a change in the vertical direction, and the time relation to the eye movement was examined. What is important in this process is that the time relation in the horizontal movement to repeatedly flashed spot light should be the same as the time relation to the case in which the spots are flashed sequentially from the top. In order to check the agreement of the time course, the following preliminary experiment was performed.

The same LED $\left(S^{*}\right)$ as was used in $S$ was placed immediately above the top LED of the light spot sequence $\mathrm{S}$, and was flashed for the same period as $\mathrm{S}$ was flashed. $\mathrm{S}$ was flashed sequentially from the top, each LED for $2 \mathrm{~ms}$, and $S^{*}$ was repeatedly flashed on and off for $2 \mathrm{~ms}$. If both ends of $\mathrm{S}$ and $\mathrm{S}^{*}$ always coincided, it was judged that the time courses of $S$ and $S^{*}$ in the horizontal direction were the same. While turning $S$ and $S^{*}$ on in various timings in perisaccade for on-times of $32,64,96$, and $128 \mathrm{~ms}$, the subject was instructed to observe the deviation at both ends.

The results show that all subjects perceived that both ends of $\mathrm{S}$ and $\mathrm{S}^{*}$ agreed. Consequently, it is considered in this experiment that the time course of PA in the horizontal direction was expanded by $\mathrm{S}$ in the vertical direction.

\subsection{Data processing}

For each of the subjects, data for the express saccade with small latency variation, consisting of 20 trials in each set, were obtained, except for the following cases. In trials of $8^{\circ}$, the case in which the saccade amplitude was below $6^{\circ}$ or above $10^{\circ}$ was excluded. In trials of $12^{\circ}$, the case in which the saccade amplitude was below $9^{\circ}$ or above $13^{\circ}$ was excluded (since the undershoot tendency was observed in all subjects in the $12^{\circ}$ trials, the lower threshold was set as $9^{\circ}$ ). In all trials, the case in which the saccade latency was below $80 \mathrm{~ms}$ or above $130 \mathrm{~ms}$ was excluded.

All images perceived in the saccade with a latency between $80 \mathrm{~ms}$ and $130 \mathrm{~ms}$ were continuations of a straight line, slant line, and straight line as shown in Fig. 6 left. Table 1 shows the saccade parameters of the subjects in this experiment. The values in the table are averages of 20 trials, and the standard deviation is shown in parentheses. An undershoot tendency is observed for the $12^{\circ}$ amplitude, but the saccade is stable in terms of both duration and latency.

The onset and offset of the saccade are defined as follows. The measured data were passed through a $100-\mathrm{Hz}$ 
Table 1. Amplitude, duration, and latency of measured saccades

\begin{tabular}{lrrrr}
\hline & Y.I. & Y.A. & R.T. & J.W. \\
\hline Amplitude [deg] & \multicolumn{1}{c}{} \\
8-Start point & $7.7(1.1)$ & $7.2(0.7)$ & $7.3(1.7)$ & $8.3(1.7)$ \\
8-End point & $7.6(0.7)$ & $8.3(1.6)$ & $6.8(1.6)$ & $7.8(1.8)$ \\
12-Start point & $9.5(0.8)$ & $11.0(1.6)$ & $9.0(1.6)$ & $11.0(1.7)$ \\
12-End point & $10.0(1.0)$ & $9.6(1.7)$ & $8.8(1.2)$ & $10.6(1.6)$ \\
\hline Duration [ms] & & & \\
8- Start point & $38.8(4.7)$ & $39.5(7.1)$ & $38.7(13.4)$ & $37.7(4.0)$ \\
8- End point & $38.5(3.1)$ & $44.5(7.3)$ & $38.0(10.9)$ & $36.5(6.5)$ \\
12-Start point 43.2(3.6) & $53.1(7.4)$ & $40.8(7.9)$ & $44.9(5.2)$ \\
12-End point $45.7(3.6)$ & $50.0(9.1)$ & $39.8(5.5)$ & $42.5(4.9)$ \\
\hline Latency [ms] & & & \\
8- Start point & $91.2(4.5)$ & $92.2(9.9)$ & $104.7(14.0)$ & $91.0(15.9)$ \\
8- End point & $99.7(7.1)$ & $96.1(11.8)$ & $93.8(20.4)$ & $95.5(10.6)$ \\
12-Start point 98.1(11.5) $101.6(12.5)$ & $105.4(17.8)$ & $87.8(19.7)$ \\
12-End point $98.8(5.8)$ & $99.8(13.5)$ & $96.0(26.6)$ & $95.0(9.9)$ \\
\hline
\end{tabular}

low-pass filter. Then, the first time at which the eye movement velocity exceeded $40 \%$ was defined as the onset of the saccade, and the last time when the eye movement velocity was above $40 \% \mathrm{~s}$ was defined as the offset of the saccade.

\subsection{Experimental results}

Table 2 shows the time relation between the start/end positions indicated by the subject and the eye movement. The top item is the time difference, obtained by subtracting the onset/offset time of the saccade (circle at right of Fig. 6) from the time when LED at the indicated start/end point is on (cross at left of Fig. 6). The positive value at the start point implies that the perceived light spot locus starts to curve after the onset of eye movement. The positive value at the end point implies that the locus returns to the straight line after the offset of the eye movement.

As regards the perception at the starting point, the perceived light spot locus starts to curve after approximately $10 \mathrm{~ms}$, as the average of four subjects, from the onset of saccade, in both the $8^{\circ}$ and $12^{\circ}$ trials. At the end point, the locus returns to the straight line in approximately $0 \mathrm{~ms}$ in the $8^{\circ}$ trial, and approximately $1 \mathrm{~ms}$ in the $12^{\circ}$ trial after the offset of saccade.

Figure 7 is a conceptual diagram based on the experimental data, the time course of the eye movement, the flash timing of the light spot array, and the timing of the start/end point of the perceived light spot locus. The vertical axis is the eye position (deg) and the horizontal axis is the time (ms). The dotted line arrow indicates the period in which the slant line is perceived, and the thick line arrow indicates
Table 2. Time gap of perceived images and measured saccades, eye movement velocity, position, width of the perceived light locus, and ratio to amplitude

\begin{tabular}{lrrrr}
\hline & Y.I. & Y.A. & R.T. & J.W. \\
\hline Time difference [ms] & & & & \\
8-Start point & 8.0 & 13.8 & 8.5 & 9.7 \\
& $(5.8)$ & $(7.2)$ & $(3.5)$ & $(6.4)$ \\
8-End point & -1.1 & -0.5 & 0.6 & 1.4 \\
& $(3.8)$ & $(6.3)$ & $(5.0)$ & $(3.0)$ \\
12-Start point & 10.0 & 6.0 & 12.1 & 11.8 \\
& $(2.1)$ & $(3.9)$ & $(5.9)$ & $(6.2)$ \\
12-End point & -2.8 & 0.9 & -2.2 & 6.5 \\
& $(4.8)$ & $(6.1)$ & $(7.3)$ & $(7.3)$ \\
\hline Eye velocity [deg/s] & & & & \\
8- Start point & 162.9 & 249.2 & 155.5 & 168.3 \\
& $(62.1)$ & $(130.0)$ & $(71.1)$ & $(63.4)$ \\
8-End point & 71.1 & 41.1 & -1.4 & 20.3 \\
& $(53.1)$ & $(62.4)$ & $(103.1)$ & $(209.7)$ \\
12-Start point & 226.9 & 159.3 & 226.9 & 136.6 \\
& $(73.4)$ & $(64.8)$ & $(104.8)$ & $(84.6)$ \\
12-End point & 94.50 & 56.62 & 46.6 & 88.2 \\
& $(51.5)$ & $(59.4)$ & $(7.2)$ & $(95.4)$ \\
\hline Position of start/end point $[\mathrm{deg}]$ & & & \\
8-Start point & 3.15 & 4.35 & 2.69 & 4.07 \\
& $(0.36)$ & $(0.73)$ & $(0.21)$ & $(0.96)$ \\
8-End point & -1.68 & -0.57 & -1.28 & -0.76 \\
& $(0.34)$ & $(0.50)$ & $(0.36)$ & $(0.66)$ \\
12-Start point & 4.92 & 6.52 & 3.23 & 4.35 \\
& $(0.50)$ & $0.96)$ & $(0.81)$ & $(0.74)$ \\
12-End point & -1.07 & -0.77 & -1.85 & -2.48 \\
& $(0.50)$ & $(0.44)$ & $(0.43)$ & $(0.81)$ \\
\hline Perceived width [deg] & & & & \\
8 deg & 4.83 & 4.92 & 3.97 & 4.83 \\
Ratio & 0.63 & 0.63 & 0.56 & 0.60 \\
12 deg & 5.99 & 7.29 & 5.08 & 6.63 \\
Ratio & 0.61 & 0.71 & 0.57 & 0.61 \\
\hline
\end{tabular}

the time difference between the saccade onset and the perception start point.

In order to assure that the above time difference is not due to pointing error of the laser pointer, the error between the perceived position and the position indicated by the laser pointer was measured. LEDs with the same intensity as the light spot array were placed in an arrangement with the same shape (left side of Fig. 6) and the same size (total height $12.8^{\circ}$, horizontal distance $5^{\circ}$ between start and end points and vertical distance $4^{\circ}$ ) as those of the actually perceived light spot locus for $8^{\circ}$ saccade, and were flashed successively from the top, each for $2 \mathrm{~ms}$. The subjects indicated the start and the end points without moving their eyes.

Table 3 shows the average error between the actually flashed start/end points and the indicated positions. The standard deviation is shown in parentheses. The first item 


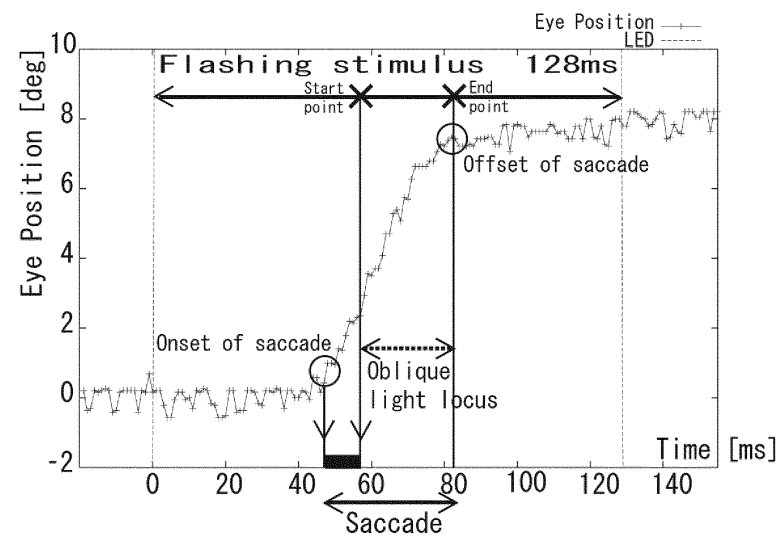

Fig. 7. Time course of eye position and light stimulus.

is the deviation represented by the view angle. The upper side is defined as positive. The second item is the time difference in the experiment, converted from the error in terms of the view angle. Thus, the sum of the first item in Table 2 and the second item in Table 3 is the time difference between the actually perceived start/end point and the eye movement. When this correction is applied, the average time difference for four subjects for $8^{\circ}$ is that the starting point is $11.3 \mathrm{~ms}$ after the onset of saccade, and the end point is $1.6 \mathrm{~ms}$ after the offset of saccade.

The second item in Table 2 is the eye movement velocity at the time when the start/end point is indicated. The start point is perceived approximately $10 \mathrm{~ms}$ after the onset of saccade. Consequently, the eye movement has already reached a velocity of 150 to $250 \%$ s. The end point, on the other hand, is perceived almost at the same time as the offset of the saccade, and there are no data exceeding $100 \%$ s.

The third item shows the indicated position of the start/end point. The position is defined as positive in the direction of the saccade, and is represented in terms of the view angle. The fourth item is the distance between the start point and the end point in the horizontal direction. It is the ratio of the horizontal width of the perceived light spot

Table 3. Error of pointing

\begin{tabular}{lrrrr}
\hline & Y.I. & Y.A. & R.T. & J.W. \\
\hline View angle [deg] & & & & \\
Start point & 0.01 & 0.07 & -0.11 & -0.36 \\
& $(0.13)$ & $(0.30)$ & $(0.42)$ & $(0.34)$ \\
End point & 0.02 & 0.12 & -0.49 & -0.30 \\
& $(0.21)$ & $(0.23)$ & $(0.46)$ & $(0.29)$ \\
\hline Time [ms] & & & & \\
Start point & 0.1 & -0.7 & 1.1 & 3.5 \\
& $(1.2)$ & $(2.9)$ & $(4.0)$ & $(3.3)$ \\
End point & -0.2 & -1.2 & 4.7 & 2.9 \\
& $(2.0)$ & $(2.2)$ & $(4.4)$ & $(2.8)$ \\
\hline
\end{tabular}

locus, that is, the width of the perceived light spot locus, to the saccade amplitude. Except for the $12^{\circ}$ trial of Y.A., the width is approximately $60 \%$ of the saccade width. For the saccade amplitude, the average value for the start and the end points in the experiment is used.

\subsection{Dependence on position of light spot sequence}

As regards the localization error for a flashed light spot, it is known that the time locus differs depending on the position of the light spot when a background is present [12]. Observing the spatial perception characteristics for the equiluminant stimulus during saccade, as examined by Ross and colleagues, the localization error reverses its sign on both sides of the target position of the saccade [13]. Thus, it can happen that the spatial perception characteristics during saccade change depending on the position of the stimulus presentation.

Consequently, the time relation between the perceived PA and the eye movement is examined in this study by varying the stimulus position of the presented light spot sequence. The experimental procedure, the device, and the flash timing of LED are the same as in the previous experiment. The difference is that the position of the light spot sequence for 128 -ms flashing is set at one of $-8^{\circ},-4^{\circ}, 4^{\circ}$, and $8^{\circ}$, not $0^{\circ}$. The time relation between the start/end point of the perceived light spot locus and the eye movement is examined.

The subjects were two of the persons (i.e., Y.I. and Y.A.) who participated in the experiment with the light spot array set at $0^{\circ}$. At any position, a light spot locus with a sigmoid function shape, as shown at the left at Fig. 6, is perceived, with the same direction of movement. In this experiment, there is an intensity difference between the light spot array and the background, and no stimulus is presented that can serve as a cue after the saccade. Consequently, the reversal of the perception direction, as reported by Ross and colleagues, was not produced [14]. The number of trials was 20 for each of the start and end points.

Table 4 shows the experimental results for the two subjects. The values in the table are the average for each item. The first item is the time difference between the onset/offset of the measured saccade and the perceived start/end point. The time difference for the start point is 7.7 to $16.8 \mathrm{~ms}$ for Y.I., and 5.5 to $13.8 \mathrm{~ms}$ for Y.A. For the end point it is -6.0 to $4.1 \mathrm{~ms}$ for Y.I., and -6.5 to $-0.5 \mathrm{~ms}$ for Y.A. Thus, both exhibited the same tendency as when the light spot array was placed at $0^{\circ}$, namely, that the start point is slightly after the onset of saccade, and the end point is almost the same as the offset of saccade. Thus, we see that the time relation between the perceived PA and the eye 
Table 4. Time gap of a perceived image and a measured saccade, position and width of the perceived light trace, and ratio to amplitude: data of Y.I. (above), Y.A. (below)

\begin{tabular}{lrrrrr}
\hline Data for Y.I. & & & & & \\
\hline & $-8 \mathrm{deg}$ & $-4 \mathrm{deg}$ & $0 \mathrm{deg}$ & $4 \mathrm{deg}$ & $8 \mathrm{deg}$ \\
\hline Time difference[ms] & & & & & \\
$\quad$ Start point & 12.2 & 7.7 & 8.0 & 16.8 & 10.5 \\
$\quad$ End point & -6.0 & -1.9 & -1.1 & 4.1 & -2.4 \\
\hline Position of start/end point [deg] & & & & \\
$\quad$ Start point & 2.34 & 3.36 & 3.15 & 2.84 & 2.79 \\
$\quad$ End point & -2.40 & -1.45 & -1.32 & -1.07 & -0.61 \\
\hline Perceived width [deg] & 4.75 & 4.81 & 4.47 & 3.91 & 3.40 \\
Ratio & 0.58 & 0.60 & 0.59 & 0.50 & 0.50 \\
\hline Data for Y.A. & & & & & \\
\hline \multicolumn{7}{l}{} & $-8 \mathrm{deg}$ & $-4 \mathrm{deg}$ & $0 \mathrm{deg}$ & $4 \mathrm{deg}$ & $8 \mathrm{deg}$ \\
\hline Time difference [ms] & & & & & \\
Start point & 12.0 & 5.5 & 13.8 & 11.4 & 8.9 \\
$\quad$ End point & -2.0 & -6.5 & -0.5 & -2.4 & -2.3 \\
\hline Position of start/end point [deg] & & & & \\
$\quad$ Start point & 5.45 & 5.63 & 4.35 & 5.89 & 7.20 \\
$\quad$ End point & 0.99 & 1.57 & -0.57 & 0.36 & 1.89 \\
\hline Perceived width [deg] & 4.46 & 5.06 & 5.53 & 4.92 & 5.31 \\
Ratio & 0.62 & 0.64 & 0.63 & 0.67 & 0.64 \\
\hline
\end{tabular}

movement is not affected by the position of the light spot array in the environment of this experiment.

The second item is the positions of the perceived start/end points. The direction of the saccade with respect to the position of the light spot array is defined as positive, and the value is represented in terms of the view angle. The third item is the perceived horizontal width and the ratio to the amplitude. In the data for Y.I., the perceived position is slightly affected. The position of the end point shifts in the saccade direction as the light spot array moves in the saccade direction, with the total width being somewhat decreased. In the data for Y.A., the position and size of the perceived light spot locus are not affected by the position of the light spot sequence.

It is generally understood that there is a large individual difference in the perception of the PA [15]. In this experiment, the individual difference is observed in the horizontal position when the position of the light spot array is modified, but there is no significant difference between individuals along the time axis.

\subsection{When the on-time of light spot array is increased}

According to the study by Honda, the localization error starts approximately $100 \mathrm{~ms}$ before the saccade and continues even approximately $100 \mathrm{~ms}$ after the saccade [7]. The localization error for flash stimulus occurs for a perisaccadic interval of approximately $250 \mathrm{~ms}$. In this experi- ment, on the other hand, the light spot array flashes from approximately $40 \mathrm{~ms}$ before the saccade to approximately $40 \mathrm{~ms}$ after the saccade. It has a period of $128 \mathrm{~ms}$, and the period of light spot flashing is shorter than the period in which the spatial localization error is produced. This makes it difficult to discuss the perception characteristics of the repeated flashing point, including all periods in which the spatial localization error is produced.

Consequently, the light spot sequence $\mathrm{S}$ was flashed successively after the saccade target is on, $4 \mathrm{~ms}$ for each spot, from the top for $256 \mathrm{~ms}$, and we investigated what light spot locus the subject perceived. We found that the light spot locus of the sigmoid function shape as in Fig. 6 was perceived in the same way as in the case of $128-\mathrm{ms}$ flashing. Since the on-time of each spot was increased, the slope of the slant line was closer to the horizontal line.

\subsection{Difference depending kind of saccade}

In this experiment, the express saccade with latency stable in time was induced in order to establish stable experimental conditions. It is known, however, that the dynamic characteristics of the saccade, such as the amplitude, maximum velocity, and duration, change with the on timing of the saccade target [16]. Consequently, the light spot array was observed by varying the on timing of the target as $0,50,100$, and $150 \mathrm{~ms}$ after vanishing of the target. However, there was no change in the shape of the perceived light spot locus.

\section{Discussion}

In this experiment, the light spot stimulus is presented in the vertical direction in perisaccadic period, and the subject is instructed to state the start point and the end point of the perceived light spot locus. The result was that the perceived light spot locus started to curve at the onset of the saccade in the opposite direction to the saccade, and returned to the vertical direction at the offset of the saccade.

Combined with the result of the preliminary experiment reported in Section 3.3, we see that the time at which the light spot stimulus flashing in the vertical direction started to curve was the start when PA is produced, and the time when it returned to the vertical direction was the end of the PA spread. Thus, in contrast to the time course expected in accordance with the cancellation theory, the PA is perceived only during the saccade (this result agrees with the results of the experiment by Oishi and Ebisawa, in which a single point was repeatedly flashed, beginning at a time prior to the saccade [2]).

As regards the direction of motion of the PA, a light spot locus curving in the opposite direction to the saccade was perceived in this experiment. This agrees with 
Hershberger's report [10]. But in the experiment on subjective observation by Oishi and Ebisawa, the PA moved in the same direction as the saccade [2]. In fact, in Hershberger's experiment too, approximately $10 \%$ of the subjects perceived that the light spot moved in the same direction as the saccade [10].

Assuming that the PA moves in the same direction as the saccade when the light spot is moved in the vertical direction as in this experiment, a light spot locus curving in the same direction as the saccade should be perceived. However, no such result was obtained in this experiment. It is conceivable that the subjectively perceived direction of motion and the actual eye movement do not necessarily agree with the time relation.

If it is assumed that the right end of the PA in Oishi and Ebisawa's experiment is not the light spot which was presented last, but the first light spot that started to spread, their results agree with the results of this experiment. The following factors might produce the different views concerning the difference in the perceived direction of motion. In Oishi and Ebisawa's experiment, the focus point and the target point were always on. Repeated flashing of the light spot was continued from $2 \mathrm{~s}$ prior to the saccade. There is also an effect of the effort to control the attention [3].

The light spot locus perceived in this experiment had the shape of the sigmoid function. The vertical straight line was perceived before and after the saccade. The reason seems to be as follows. In the perception of the repeatedly flashing light spot, the image of the light spot which was on immediately before exists on the retina as a cue for localization. Consequently, localization is performed on the basis of the relative position information on the retina before and after the saccade, where the eye is still [17].

Furthermore, the start of the observed light spot locus was perceived as being displaced in the same direction as the saccade, and the end was perceived as being displaced in the opposite direction to the saccade. The reason for the mechanism that produces such a perception may be as follows. During the saccade, in which the retinal information changes rapidly, the absolute spatial localization is performed in accordance with the cancellation theory, while referring to the eye position information. Then, the position of the light spot before and after the saccade is reconstructed on the basis of the relative position relation, with the above cue as the reference. However, another mechanism may be considered in which the velocity perception in the horizontal direction is reduced during saccade, and an image smaller than the horizontal movement on the retina is perceived [18], and the localization is performed with those as a set of images [19].

In this experiment, the start of the perceived light spot locus is approximately $10 \mathrm{~ms}$ later than the onset of the saccade. The reason seems to be as follows. The spatial localization mechanism cannot be switched quickly. Thus, the information on the retina is located with an error in the opposite direction to the saccade. But when the localization is based on the cancellation theory, the error is in the same direction as the saccade. These are canceled at the time of switching. In addition to such a factor related to the mechanism, the perception of the velocity in the vertical direction may be affected in the saccade, as indicated in the report that the velocity perception after the saccade is enhanced in the direction opposite to the saccade [20].

\section{Conclusions}

This paper has analyzed the time relation of the perceived locus for the repeatedly flashed light spot in the perisaccadic period to the eye movement, by successively turning on the light spot array in the vertical direction. This is a point that has not been analyzed in the experiment of Hershberger and colleagues.

This experiment showed that the time for which the information can be presented in SD is slightly shorter than the duration of the saccade. The direction in which the two-dimensional image spreads is opposite to the saccade, and the horizontal length for which the information can be presented is approximately $60 \%$ of the saccade amplitude.

Acknowledgments. The authors are grateful for useful advice given in this study by Mr. M. Imai, The University of Tokyo, and Mr. A. Noritake, Kwansei Gakuin University.

\section{REFERENCES}

1. Watanabe J, Maeda T, Tachi S. The study of saccadebased display. J VR Soc Jpn 2001;6:79-87.

2. Oishi S, Ebisawa Y. Influence of attended direction upon voluntary saccade-contingent visual mislocalization of a repeatedly flashing stimulus. J Inst Image Inform Telev Eng 2002;56:1825-1829.

3. McFadden SA, Khan A, Wallman J. Gain adaptation of exogenous shifts of visual attention. Vision Res 2002;42:2709-2726.

4. Oyama T, Imai S, Wake T (New Ed.). Handbook for psychology of sensation and perception. Senshin Shobo; 1993.

5. Burr DC, Morrone MC, Ross J. Selective suppression of the magnocellular visual pathway during saccadic eye movements. Nature 1994;371:511-513.

6. Fujita M, Amami A. Iconic memory during human saccadic and fast smooth pursuit eye movements (1). Tech Rep IEICE 2000;NC2000-41. 
7. Honda H. Eye movements to a visual stimulus flashed before, during, or after a saccade. Attention and Performance 1990;13:567-582.

8. Honda H. Eye movement and spatial localization. Kazama Shobo; 1994.

9. Hershberger WA. Saccadic eye movements and the perception of visual direction. Percept Psychophys 1987;41:35-44.

10. Hershberger WA, Jordan JS, Lucas DR. Visualization the perisaccadic shift of spatiotopic coordinates. Percept Psychophys 1998;60:82-88.

11. Fischer B, Ramsperger E. Human express saccades: Extremely short reaction times of goal directed eye movements. Exp Brain Res 1984;57:191-195.

12. Honda H. Saccade-contingent displacement of the apparent position of visual stimuli flashed on a dimly illuminated structured background. Vision Res 1993;33:709-716.

13. Ross J, Morrone MC, Burr DC. Compression of visual space before saccades. Nature 1997;386:598601.

14. Lappe M, Awater H, Krekelberg B. Postsaccadeic visual references generate presaccadic compression of space. Nature 2000;403:892-895.
15. Hershberger WA, Jordan JS. Visual direction constancy: Perceiving the visual direction of perisaccadic flashes. In Chekaluk E, Llewellyn KR (editors). The role of eye movements in perceptual processes. Elsevier; 1992. p 1-43.

16. Aoki M, Fujita M. Adaptive transfer between scanning saccade and memory-guided saccade. Trans IEICE 1999;J82-D-II:91-99.

17. Sogo H, Osaka N. Perception of relation of stimuli locations successively flashed before saccade. Vision Res 2001;41:935-942.

18. Bedell HE, Yang J. The attenuation of perceived image smear during saccades. Vision Res 2001;41:521-528.

19. Matsumiya K, Uchikawa K. Apparent size of an object remains uncompressed during presaccadic compression of visual space. Vision Res 2001;41:3039-3050.

20. Park J, Lee J, Lee C. Non-veridical visual motion perception immediately after saccades. Vision Res 2001;41:3751-3761.

AUTHORS (from left to right)
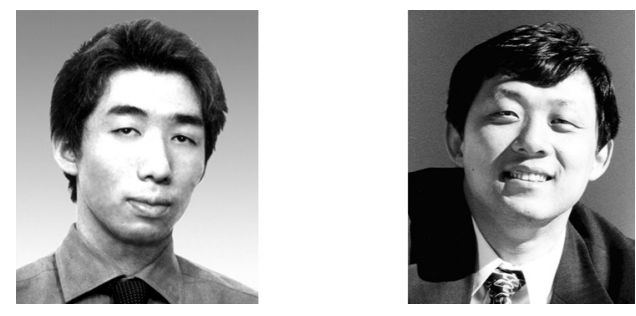

Junji Watanabe received his B.S. degree from the Department of Mathematical Engineering and Information Physics, The University of Tokyo, in 2000, completed the M.E. program in 2002, and is now a doctoral candidate. His research concerns displays based on eye movement and visual characteristics during eye movement. He is a member of the VR Society of Japan, The Vision Society of Japan, The Institute of Image Information and Television Engineering, and Human Interface Society. He has received a VR Society of Japan Paper Award (2001) and Encouragement Award (2002).

Taro Maeda (member) received his B.S. degree from the Department of Mathematical Engineering and Information Physics, The University of Tokyo, in 1987 and joined the Mechanical Engineering Laboratory, MITI. He moved to The University of Tokyo in 1992, and has been a lecturer in the Graduate School of Interdisciplinary Information Studies since 2000. His research interests are modeling of human perception characteristics and neural network, as well as tele-existence. He has received a Society of Instrument and Control Engineers Paper Award and Encouragement Award, and Technology Award from the Robotics Society of Japan. 
AUTHORS (continued)

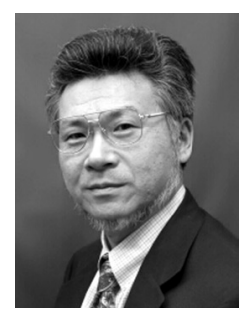

Susumu Tachi (member) received his B.S. degree from the Department of Mathematical Engineering and Information Physics, The University of Tokyo, in 1968 and completed the doctoral program in 1973, receiving a D.Eng. degree. After becoming a professor at the Research Center for Advanced Science and Technology (1992) and the Department of Mathematical Engineering and Information Physics (1994), he has been a professor of information physics and computing in the Graduate School of Information Science and Technology since 2001. His research interests are tele-existence and virtual reality. He has received an IEEE/EMBS Award, MITI Minister's Award, and Special Achievement Award from the International Measurement Confederation. He served as Chairman of the IMEKO Robotics Conference and is a past president of the VR Society of Japan. He is an SICE fellow. 